\title{
ROTATING CHARGED BLACK HOLE SOLUTION IN HETEROTIC STRING THEORY
}

\author{
Ashoke Sen \\ Tata Institute of Fundamental Research \\ Homi Bhabha Road, Bombay 400005, India \\ e-mail address: sen@tifrvax.bitnet
}

\begin{abstract}
We construct a solution of the classical equations of motion arising in the low energy effective field theory for heterotic string theory. This solution describes a black hole in four dimensions carrying mass $M$, charge $Q$ and angular momentum $J$. The extremal limit of the solution is discussed.
\end{abstract}


It has been realized recently that the low energy effective field theory describing string theory contains black hole (or, more generally, black $p$-brane) solutions which can have properties which are qualitatively different from those that appear in ordinary Einstein gravity[1]. Most of these solutions are characterized by one or more charges associated with Yang-Mills fields or the antisymmetric tensor gauge field, and a non-trivial dilaton field. In the absence of any charge, the solution reduces to the ordinary Schwarzschild solution.

Rotating charge neutral black hole solutions can also be constructed in string theory, and are identical to the Kerr solution[2] of ordinary Einstein gravity with the dilaton taking a constant value. Recently, rotating charged black hole solutions in these theories have been analyzed[3] in the limit of small angular momentum. In fact, in ref.[3] the authors consider a more general class of theories than those which arise as the low energy effective action in string theory, by allowing dilaton couplings to the Maxwell field of the type which is not necessarily the one induced in string theory. They, however, consider only the dilatongraviton system, and do not consider more general action that also includes antisymmetric tensor gauge field.

In this paper we shall construct an exact classical solution in the low energy effective field theory describing heterotic string theory, which describes a black hole carrying finite amount of charge and angular momentum. Our solution, however, differs from that of ref.[3] even in the limit of small angular momentum since it involves the antisymmetric tensor field in a non-trivial way. In fact, since a rotating charged black hole also carries a magnetic dipole moment, the antisymmetric tensor field background is induced automatically if we take into account the coupling of the antisymmetric tensor gauge field strength to the Chern-Simons three form constructed from the gauge fields.

The method that we shall be using for obtaining the solution is the twisting procedure[4]- 
[8] that generates inequivalent classical solutions starting from a given classical solution of string theory. In particular, in ref.[7] it was shown how one can generate charged blackhole solutions starting from a charge neutral solution. With the help of the same transformations, we shall generate the rotating charged black hole solution by starting from a rotating black hole solution carrying no charge, i.e. the Kerr solution[2].

We shall first summarize the results of ref.[7] applied to the particular problem at hand. We begin with the string theory effective action in four dimensions:

$$
S=-\int d^{4} x \sqrt{-\operatorname{det} G} e^{-\Phi}\left(-R+\frac{1}{12} H_{\mu \nu \rho} H^{\mu \nu \rho}-G^{\mu \nu} \partial_{\mu} \Phi \partial_{\nu} \Phi+\frac{1}{8} F_{\mu \nu} F^{\mu \nu}\right)
$$

Here $G_{\mu \nu}$ is the metric, $R$ is the scalar curvature, $F_{\mu \nu}=\partial_{\mu} A_{\nu}-\partial_{\nu} A_{\mu}$ is the field strength corresponding to the Maxwell field $A_{\mu}, \Phi$ is the dilaton field, and,

$$
H_{\mu \nu \rho}=\partial_{\mu} B_{\nu \rho}+\text { cyclic permutations }-\left(\Omega_{3}(A)\right)_{\mu \nu \rho}
$$

where $B_{\mu \nu}$ is the antisymmetric tensor gauge field, and,

$$
\left(\Omega_{3}(A)\right)_{\mu \nu \rho}=\frac{1}{4}\left(A_{\mu} F_{\nu \rho}+\text { cyclic permutations }\right)
$$

is the gauge Chern-Simons term. There are several points we need to mention at this stage. They are the followings.

1. We are considering a theory where 6 of the 10 dimensions have been compactified (say, to a Calabi-Yau manifold). The massless fields arising from compactification have not been included in the effective action.

2. We have included only a $U(1)$ component of the full set of non-abelian gauge fields present in the theory. This will suffice for our purpose, since we shall look for solutions carrying $U(1)$ charge only.

3. The metric $G_{\mu \nu}$ used here is the metric that arises naturally in the $\sigma$-model, and is related to the Einstein metric $G_{\mu \nu}^{(E)}$ through the relation:

$$
G_{\mu \nu}^{(E)}=e^{-\Phi} G_{\mu \nu}
$$


After this field redefinition (together with a rescaling $\Phi \rightarrow 2 \Phi, A_{\mu} \rightarrow 2 \sqrt{2} A_{\mu}$ ) one can recover the action of ref.[3], except for the $H_{\mu \nu \rho} H^{\mu \nu \rho}$ term appearing in eq.(1).

4. We have truncated the action to contain only those terms that contain two or less number of derivatives. Thus, for example, the Lorentz Chern Simons term has not been included in the definition of $H_{\mu \nu \rho}$, since the corresponding terms in the action will contain more than two derivatives.

We shall look for solutions that are independent of the time coordinate $t$. In the following analysis we shall use matrix notation to describe the various fields. In this notation, $G_{\mu \nu}$ and $B_{\mu \nu}$ will be treated as $4 \times 4$ matrices, and $A_{\mu}$ will be treated as a four dimensional column vector, with the fourth row and/or column corresponding to the time coordinate $t$. Let us now define the matrices $\mathcal{K}, \eta$ and $\mathcal{M}$ as,

$$
\begin{gathered}
\mathcal{K}_{\mu \nu}=-B_{\mu \nu}-G_{\mu \nu}-\frac{1}{4} A_{\mu} A_{\nu} \\
\eta_{\mu \nu}=\operatorname{Diag}(1,1,1,-1)
\end{gathered}
$$

and

$$
\mathcal{M}=\left(\begin{array}{ccc}
\left(\mathcal{K}^{T}-\eta\right) G^{-1}(\mathcal{K}-\eta) & \left(\mathcal{K}^{T}-\eta\right) G^{-1}(\mathcal{K}+\eta) & -\left(\mathcal{K}^{T}-\eta\right) G^{-1} A \\
\left(\mathcal{K}^{T}+\eta\right) G^{-1}(\mathcal{K}-\eta) & \left(\mathcal{K}^{T}+\eta\right) G^{-1}(\mathcal{K}+\eta) & -\left(\mathcal{K}^{T}+\eta\right) G^{-1} A \\
-A^{T} G^{-1}(\mathcal{K}-\eta) & -A^{T} G^{-1}(\mathcal{K}+\eta) & A^{T} G^{-1} A
\end{array}\right)
$$

Here $T$ denotes transposition of a matrix. Eq.(7) defines a $9 \times 9$ matrix $\mathcal{M}$. The result of ref.[7] then says that if $\left\{G_{\mu \nu}, B_{\mu \nu}, \Phi, A_{\mu}\right\}$ describes a time independent solution of the classical equations of motion derived from the action given in eq.(1), then $\left\{G_{\mu \nu}^{\prime}, B_{\mu \nu}^{\prime}, \Phi^{\prime}, A_{\mu}^{\prime}\right\}$ also describes a solution of the same equations of motion, if the primed variables are related to the unprimed ones through the relation,

$$
\mathcal{M}^{\prime}=\Omega \mathcal{M} \Omega^{T}, \quad \Phi^{\prime}-\ln \operatorname{det} G^{\prime}=\Phi-\ln \operatorname{det} G
$$

where,

$$
\Omega=\left(\begin{array}{ccc}
I_{7} & & \\
& \cosh \alpha & \sinh \alpha \\
& \sinh \alpha & \cosh \alpha
\end{array}\right)
$$


Here $I_{7}$ denotes a $7 \times 7$ identity matrix, and $\alpha$ is an arbitrary number. Eqs.(8) uniquely determine all the primed fields in terms of the unprimed ones.

We now apply this transformation to the charge neutral rotating black hole solution. This is given by the standard Kerr solution[2]

$$
\begin{aligned}
d s^{2}= & -\frac{\rho^{2}+a^{2} \cos ^{2} \theta-2 m \rho}{\rho^{2}+a^{2} \cos ^{2} \theta} d t^{2}+\frac{\rho^{2}+a^{2} \cos ^{2} \theta}{\rho^{2}+a^{2}-2 m \rho} d \rho^{2}+\left(\rho^{2}+a^{2} \cos ^{2} \theta\right) d \theta^{2} \\
& +\frac{\sin ^{2} \theta}{\rho^{2}+a^{2} \cos ^{2} \theta}\left\{\left(\rho^{2}+a^{2}\right)\left(\rho^{2}+a^{2} \cos ^{2} \theta\right)+2 m \rho a^{2} \sin ^{2} \theta\right\} d \phi^{2}-\frac{4 m \rho a \sin ^{2} \theta}{\rho^{2}+a^{2} \cos ^{2} \theta} d t d \phi \\
\Phi= & 0, \quad B_{\mu \nu}=0, \quad A_{\mu}=0
\end{aligned}
$$

The transformed solution is given by,

$$
\begin{aligned}
& d s^{\prime 2}=-\frac{\left(\rho^{2}+a^{2} \cos ^{2} \theta-2 m \rho\right)\left(\rho^{2}+a^{2} \cos ^{2} \theta\right)}{\left(\rho^{2}+a^{2} \cos ^{2} \theta+2 m \rho \sinh ^{2} \frac{\alpha}{2}\right)^{2}} d t^{2} \\
&+\frac{\rho^{2}+a^{2} \cos ^{2} \theta}{\rho^{2}+a^{2}-2 m \rho} d \rho^{2}+\left(\rho^{2}+a^{2} \cos ^{2} \theta\right) d \theta^{2} \\
&+\left\{\left(\rho^{2}+a^{2}\right)\left(\rho^{2}+a^{2} \cos ^{2} \theta\right)+2 m \rho a^{2} \sin ^{2} \theta+4 m \rho\left(\rho^{2}+a^{2}\right) \sinh ^{2} \frac{\alpha}{2}+4 m^{2} \rho^{2} \sinh ^{4} \frac{\alpha}{2}\right\} \\
& \times \frac{\left(\rho^{2}+a^{2} \cos ^{2} \theta\right) \sin ^{2} \theta}{\left(\rho^{2}+a^{2} \cos ^{2} \theta+2 m \rho \sinh ^{2} \frac{\alpha}{2}\right)^{2}} d \phi^{2} \\
&-\frac{4 m \rho a \cosh ^{2} \frac{\alpha}{2}\left(\rho^{2}+a^{2} \cos ^{2} \theta\right) \sin ^{2} \theta}{\left(\rho^{2}+a^{2} \cos ^{2} \theta+2 m \rho \sinh ^{2} \frac{\alpha}{2}\right)^{2} d t d \phi} \\
& \Phi^{\prime}=-\ln \frac{\rho^{2}+a^{2} \cos ^{2} \theta+2 m \rho \sinh ^{2} \frac{\alpha}{2}}{\rho^{2}+a^{2} \cos ^{2} \theta} \\
& A_{\phi}^{\prime}=-\frac{2 m \rho a \sinh \alpha \sin ^{2} \theta}{\rho^{2}+a^{2} \cos ^{2} \theta+2 m \rho \sinh ^{2} \frac{\alpha}{2}} \\
& B_{t \phi}^{\prime}=\frac{2 m \rho a \sinh \frac{\alpha}{2} \sin ^{2} \theta}{\rho^{2}+a^{2} \cos ^{2} \theta+2 m \rho \sinh ^{2} \frac{\alpha}{2}} \\
& A_{t}^{\prime}=\frac{2 m \rho \sinh \alpha}{\rho^{2}+a^{2} \cos ^{2} \theta+2 m \rho \sinh ^{2} \frac{\alpha}{2}}
\end{aligned}
$$


The other components of $A_{\mu}^{\prime}$ and $B_{\mu \nu}^{\prime}$ vanish. The Einstein metric $d s_{E}^{\prime 2} \equiv e^{-\Phi^{\prime}} d s^{\prime 2}$ is given by,

$$
\begin{aligned}
d s_{E}^{\prime 2}= & -\frac{\rho^{2}+a^{2} \cos ^{2} \theta-2 m \rho}{\rho^{2}+a^{2} \cos ^{2} \theta+2 m \rho \sinh ^{2} \frac{\alpha}{2}} d t^{2}+\frac{\rho^{2}+a^{2} \cos ^{2} \theta+2 m \rho \sinh ^{2} \frac{\alpha}{2}}{\rho^{2}+a^{2}-2 m \rho} d \rho^{2} \\
& +\left(\rho^{2}+a^{2} \cos ^{2} \theta+2 m \rho \sinh ^{2} \frac{\alpha}{2}\right) d \theta^{2}-\frac{4 m \rho a \cosh ^{2} \frac{\alpha}{2} \sin ^{2} \theta}{\rho^{2}+a^{2} \cos ^{2} \theta+2 m \rho \sinh ^{2} \frac{\alpha}{2}} d t d \phi \\
& +\left\{\left(\rho^{2}+a^{2}\right)\left(\rho^{2}+a^{2} \cos ^{2} \theta\right)+2 m \rho a^{2} \sin ^{2} \theta+4 m \rho\left(\rho^{2}+a^{2}\right) \sinh ^{2} \frac{\alpha}{2}+4 m^{2} \rho^{2} \sinh ^{4} \frac{\alpha}{2}\right\} \\
& \times \frac{\sin ^{2} \theta}{\rho^{2}+a^{2} \cos ^{2} \theta+2 m \rho \sinh ^{2} \frac{\alpha}{2}} d \phi^{2}
\end{aligned}
$$

This metric describes a black hole solution with mass $M$, charge $Q$, angular momentum $J$, and magnetic dipole moment $\mu$ given by,

$$
M=\frac{m}{2}(1+\cosh \alpha), \quad Q=\frac{m}{\sqrt{2}} \sinh \alpha, \quad J=\frac{m a}{2}(1+\cosh \alpha), \quad \mu=\frac{1}{\sqrt{2}} m a \sinh \alpha
$$

so that the $g$-factor[3] is given by,

$$
g \equiv \frac{2 \mu M}{Q J}=2
$$

We shall now analyze various properties of this solution, and also discuss its extremal limit. For this purpose, it will be more convenient to express $m, a$ and $\alpha$ in terms of the independent physical parameters $M, J$ and $Q$ by inverting the relations given in eq.(17). We get,

$$
m=M-\frac{Q^{2}}{2 M}, \quad \sinh \alpha=\frac{2 \sqrt{2} Q M}{2 M^{2}-Q^{2}}, \quad a=\frac{J}{M}
$$

The coordinate singularities (horizon) occur on the surfaces

$$
\rho^{2}-2 m \rho+a^{2}=0
$$

which gives,

$$
\rho=m \pm \sqrt{m^{2}-a^{2}}=M-\frac{Q^{2}}{2 M} \pm \sqrt{\left(M-\frac{Q^{2}}{2 M}\right)^{2}-\frac{J^{2}}{M^{2}}} \equiv \rho_{H}^{ \pm}
$$


The area of the outer event horizon with the metric given in eq.(16) is given by,

$$
A=8 \pi M\left(M-\frac{Q^{2}}{2 M}+\sqrt{\left(M-\frac{Q^{2}}{2 M}\right)^{2}-\frac{J^{2}}{M^{2}}}\right)
$$

From eq.(21) we see that the horizon disappears unless,

$$
|J| \leq M^{2}-\frac{Q^{2}}{2}
$$

Thus the extremal limit of the black hole corresponds to $|J| \rightarrow M-\frac{Q^{2}}{2 M}$. In this limit, $A \rightarrow 8 \pi|J|$. Hence the event horizon remains to be of finite size in this limit, as is expected from the general arguments of ref.[3]. Note the amusing result that in the extremal limit the area of the event horizon depends only on the angular momentum $J$. Surprisingly, this result is identical to the corresponding result for the rotating charged black hole in a different model discussed in ref.[9].

The angular velocity $\Omega$ at the horizon is determined by demanding that the Killing vector $\frac{\partial}{\partial t}+\Omega \frac{\partial}{\partial \phi}$ is null at the horizon[2][3]. In other words,

$$
G_{t t}+2 G_{t \phi} \Omega+G_{\phi \phi} \Omega^{2}=0
$$

This gives,

$$
\Omega=\frac{J}{2 M^{2}} \frac{1}{M-\frac{Q^{2}}{2 M}+\sqrt{\left(M-\frac{Q^{2}}{2 M}\right)^{2}-\frac{J^{2}}{M^{2}}}}
$$

As we approach the extremal limit, $\Omega \rightarrow \frac{1}{2 M} \operatorname{sign}(J)$ as long as $|J| \neq 0$. If $J=0$, then $\Omega$ vanishes, as can be directly seen from eq.(25). It is interesting to note that in the extremal limit, $|\Omega|$ depends only on the mass of the black hole.

Finally, the surface gravity $\kappa$ (or the Hawking temperature $T_{H}=\kappa / 2 \pi$ ) is calculated at the pole as,

$$
\kappa=\left.\lim _{\rho \rightarrow \rho_{H}^{+}} \sqrt{g^{\rho \rho}} \partial_{\rho} \sqrt{-g_{t t}}\right|_{\theta=0}=\frac{\sqrt{\left(2 M^{2}-Q^{2}\right)^{2}-4 J^{2}}}{2 M\left(2 M^{2}-Q^{2}+\sqrt{\left(2 M^{2}-Q^{2}\right)^{2}-4 J^{2}}\right)}
$$


Thus in the extremal limit $\kappa \rightarrow 0$ if $J \neq 0$. On the other hand, if $J=0$, then $\kappa=\frac{1}{4 M}$, in agreement with the results of refs.[1].

To summarize, in this paper we have constructed a rotating charged black hole solution in four dimensional heterotic string theory and studied its various properties. The extremal limit of the solution was also discussed, and, for $J \neq 0$, was found to have features that are qualitatively similar to the extremal rotating black hole rather than extremal charged black hole, as was conjectured in ref.[3].

\section{References}

1. G. Gibbons and K. Maeda, Nucl. Phys. B298, 741 (1988); D. Garfinkle, G. Horowitz and A. Strominger, Phys. Rev. D43, 3140 (1991); G. Horowitz and A. Strominger, Nucl. Phys. B360, 197 (1991); A. Shapere, S. Trivedi and F. Wilczek, Mod. Phys. Lett. A6, 2677 (1991).

2. R. Adler, M. Bazin and M. Schiffer, Introduction to General Relativity, McGraw Hill, 1975; K. Thorne, R. Price and D. Macdonald, Black Holes: The Membrane Paradigm, Yale Univ. Press, 1986.

3. J. Horne and G. Horowitz, preprint UCSBTH-92-11.

4. S. Ferrara, J. Scherk and B. Zumino, Nucl. Phys. B121, 393 (1977); E. Cremmer, J. Scherk and S. Ferrara, Phys. Lett. B68, 234 (1977); B74, 61 (1978); E. Cremmer and J. Scherk, Nucl. Phys. B127, 259 (1977); E. Cremmer and B. Julia, Nucl. Phys.B159, 141 (1979); M. De Roo, Nucl. Phys. B255, 515 (1985); Phys. Lett. B156, 331 (1985);

E. Bergshoef, I.G. Koh and E. Sezgin, Phys. Lett. B155, 331 (1985); M. De Roo and 
P. Wagemans, Nucl. Phys. B262, 646 (1985); L. Castellani, A. Ceresole, S. Ferrara, R. D'Auria, P. Fre and E. Maina, Nucl. Phys. B268, 317 (1986); Phys. Lett. B161, 91 (1985); S. Cecotti, S. Ferrara and L. Girardello, Nucl. Phys. B308, 436 (1988); M. Duff, Nucl. Phys. B335, 610 (1990).

5. G. Veneziano, Phys. Lett. B265, 287 (1991); K. Meissner and G. Veneziano, Phys. Lett. B267, 33 (1991); Mod. Phys. Lett. A6, 3397 (1991); M. Gasperini, J. Maharana and G. Veneziano, Phys. Lett. B272, 277 (1991); M. Gasperini and G. Veneziano, preprint CERN-TH-6321-91.

6. A. Sen, Phys. Lett. B271, 295 (1991); 274, 34 (1991).

7. S.F. Hassan and A. Sen, preprint TIFR/TH/91-40 (to appear in Nucl. Phys. B).

8. J. Horne, G. Horowitz and A. Steif, Phys. Rev. Lett. 68, 568 (1992); M. Rocek and E. Verlinde, preprint IASSNS-HEP-91-68; P. Horava, preprint EFI-91-57; A. Giveon and M. Rocek, preprint IASSNS-HEP-91-84; S. Kar, S. Khastagir and A. Kumar, preprint IP-BBSR-91-51; J. Panvel, preprint LTH 282.

9. V. Frolov, A. Zelnikov and U. Bleyer, Ann. Phys. (Leipzig) 44, 371 (1987). 